\title{
Microstructural Characterization of Silver Nanoparticles for Bioimaging Applications
}

\author{
A. P. Zaderenko*, C. Caro*, M. de la Mata**, J. A. Sánchez** and M. J. Sayagués*** \\ * Department of Physical, Chemical and Natural Systems, University Pablo de Olavide, 41013- \\ Seville, Spain \\ ** Andalusian Center for Developmental Biology (CABD), 41013-Seville, Spain \\ *** Institute of Materials Science (ICMS), 41092-Seville, Spain
}

Silver nanoparticles are emerging as a powerful tool in bioimaging applications owing to their unique plasmonic properties i.e., extremely high molar extinction coefficients, resonant Rayleigh scattering and enhanced local electromagnetic fields [1]. Through the optimization of these properties, by controlling composition, size, shape, and interparticle spacing of nanoparticles and their assemblies [2], highly enhanced local electromagnetic fields in the vicinity of nanoparticles are achievable giving rise to IR, Raman and fluorescence surface enhanced spectroscopies (SEIRS, SERS and MEF, respectively).

In our laboratories we have synthesized and characterized by electron microscopy silver nanoparticles functionalized with the organic fluorophore Rhodamine 6G (R6G). We will be presenting data from two types of R6G silver nanoparticles that demonstrate their use as imaging agents. Nanoparticles were synthesized by reduction of silver nitrate with sodium borohydride in aqueous medium, and functionalized with R6G, as MEF probe, and polyvinylpyrrolidone (PVP), as protecting ligand. SN1 nanoparticles were functionalized during the synthesis process, by adding both R6G and PVP to the reduction medium. SN2 nanoparticles were partially aggregated after reduction, by addition of R6G, and the so obtained nanoclusters protected against further aggregation by addition of PVP. Raman characterization of SN1 and SN2 clearly confirmed the functionalization of the nanoparticles with R6G.

In vitro release of R6G from nanoparticles was studied by dialysis against water during one week. SN1 was stable under dialysis conditions while SN2 showed a sustained liberation profile. This fact is indicative of a strong linkage of R6G to silver in SN1 nanoparticles. Additionally, colloidal aqueous solutions of SN1 are stable while SN2 aggregate within hours. Microstructural characterization was carried out by using a TEM microscope (Philips CM200). Droplets of the nanoparticles suspension were deposited onto a C film grid. The obtained results for SN1 sample (Figure 1 left) show very isolated silver nanospheres with an average particle size of $20 \mathrm{~nm}$. However SN2 sample (Figure 1 center) is formed by small aggregates of silver nanoparticles with a larger particle size of approximately $50 \mathrm{~nm}$. In both cases ED analysis was performed and the ring ED patterns could be indexed as crystalline metallic silver with cubic symmetry and Fm $3 \mathrm{~m}$ space group $(\mathrm{a}=4.0862 \AA)$.

SN1 and SN2 were conjugated with monoclonal antibody to epidermal growth factor receptor (mAbEGFR; labelled with Alexa Fluor ${ }^{\circledR}$ 488), highly expressed on non-small cell lung cancer cells, through carbodiimide-mediated coupling reactions. Non-small lung cancer cell line cultures $(\mathrm{H}-460)$ were incubated with mAb-EGFR conjugated nanoparticles, and cellular uptake and bioimaging capability assessed by fluorescence microscopy (Figure 2). According to our results, SN1 and SN2 provide efficient and targeted imaging systems.

\section{References}

1. Haes A. J. and Van Duyne R. P., Anal Bioanal Chem 379: 920-930, 2004

2. Caro C., Castillo P.M., Klippstein R., Pozo D. and Zaderenko A.P., Silver nanoparticles: Sensing and imaging applications, in Silver Nanoparticles, 2010. ISBN 978-953-307-028-5 

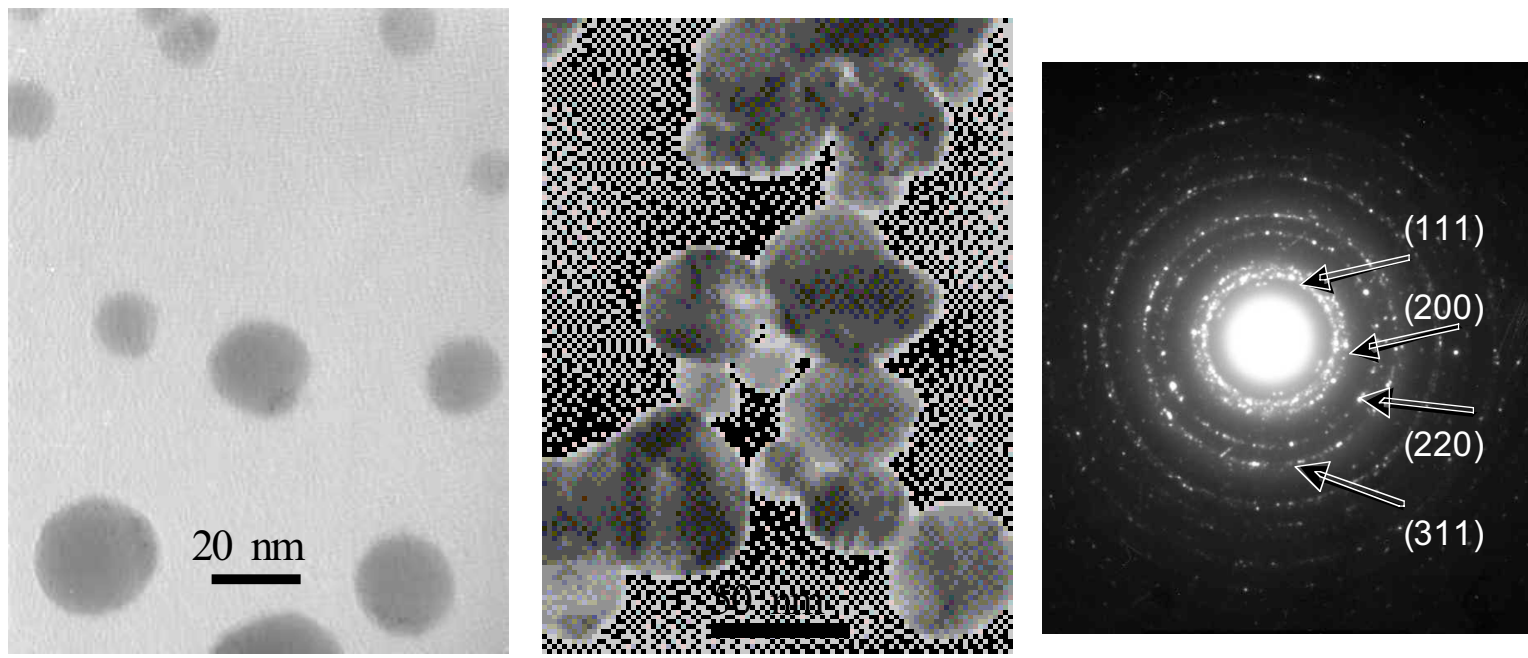

Figure 1. Microstructural characterization of SN1 and SN2.
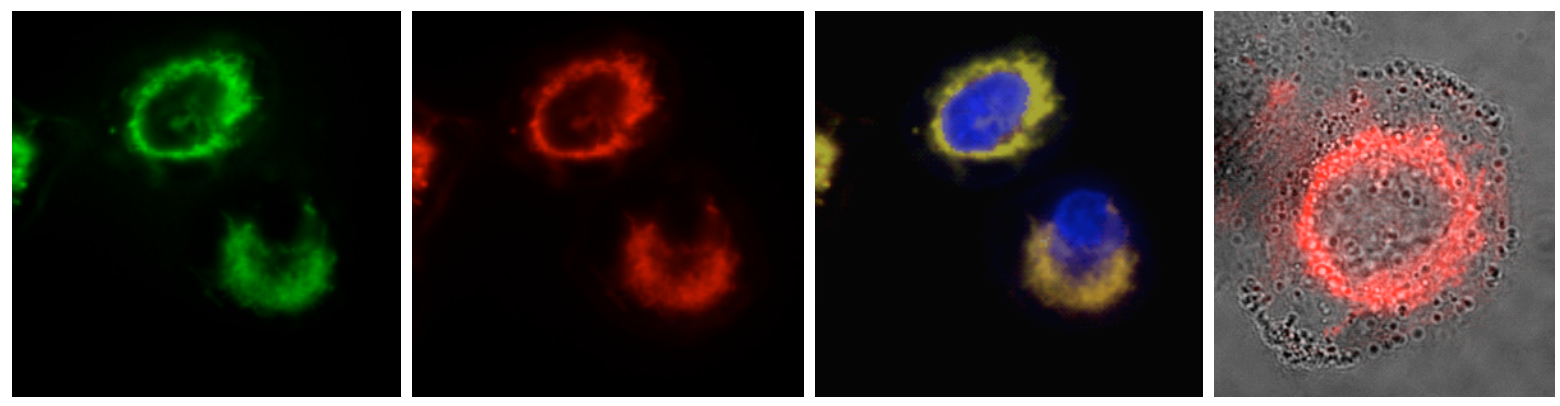

Figure 2. H-460 cultures incubated with EGFR antibody conjugated SN2 (A-C; Delta Vision system) and SN1 (D; Upright Fluorescence Microscopy Zeiss Axio Imager M2) nanoparticles. Image A corresponds to emission of mAb-EGFR conjugated to SN2 nanoparticles, image B to emission of $\mathrm{R} 6 \mathrm{G}$, and image $\mathrm{C}$ is a merge of both. Image D corresponds to emission of R6G in SN1 nanoparticles.

The authors thank financial support from the Junta de Andalucía (FQM-6615, PI0070 and FQM319). 